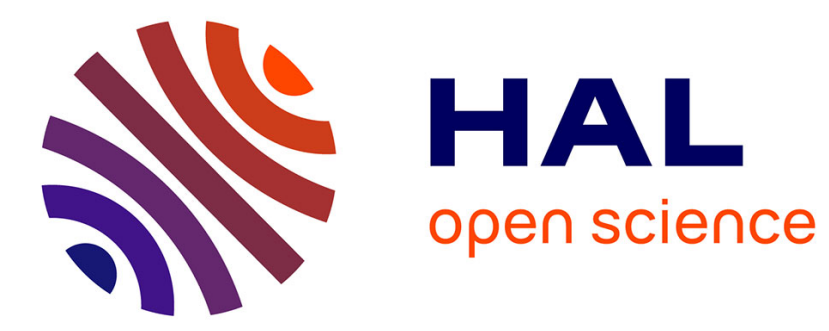

\title{
Beacon delivery over practical V2X channels
}

Oyunchimeg Shagdar, Alex Daniel, Serguei Primak

\section{To cite this version:}

Oyunchimeg Shagdar, Alex Daniel, Serguei Primak. Beacon delivery over practical V2X channels. International Conference on ITS Telecommunications, Nov 2013, Tampare, Finland. hal-00868063

\section{HAL Id: hal-00868063 https://hal.inria.fr/hal-00868063}

Submitted on 1 Oct 2013

HAL is a multi-disciplinary open access archive for the deposit and dissemination of scientific research documents, whether they are published or not. The documents may come from teaching and research institutions in France or abroad, or from public or private research centers.
L'archive ouverte pluridisciplinaire HAL, est destinée au dépôt et à la diffusion de documents scientifiques de niveau recherche, publiés ou non, émanant des établissements d'enseignement et de recherche français ou étrangers, des laboratoires publics ou privés. 


\section{Beacon delivery over practical V2X channels}

\author{
Oyunchimeg Shagdar \\ IMARA Project-team, INRIA Rocquencourt \\ Domaine de Voluceau \\ B.P. 10578153 Le Chesnay, FRANCE \\ Email: oyunchimeg.shagdar@inria.fr
}

\author{
Alex Daniel and Serguei Primak \\ innovation Centre for Information Engineering \\ Dept. of ECE, Western University \\ London, ON, N6G 5E8, CANADA \\ amdanie19@gmail.com,slprimak@uwo.ca
}

\begin{abstract}
The problem of modelling V2X system based on partial (RSSI) measurements of the wireless propagation channel is considered. The study shows that the dual slope linear model well approximates pathloss in the V2X systems. We also show that in addition to distance dependent pathloss, incorporation of fast fading as well as its frequency selectivity have significant effect on the overall performance of the system. Interference from simultaneous transmissions is estimated based on medium access control (MAC) and realistic road traffic models. Our simulation results show how car traffic parameters and MAC behavior provide direct impact on the effective communication range of the V2X system.
\end{abstract}

\section{INTRODUCTION}

Vehicular communications have gained prominent attention due to their potential for improved road safety [1]. To support V2V and V2I communications, the IEEE specified the $802.11 \mathrm{p}$ amendment for the $5.9 \mathrm{GHz}$ frequency band [2]. The road safety applications often require individual road users (vehicles) to periodically broadcast (beaconing) information on their motion status. Due to its stringent requirements in terms of information delivery and latency, beacons are expected to be transmitted in a so-called control channel $(\mathrm{CCH})$, which is a frequency channel in the $5.9 \mathrm{GHz}$ band dedicated to safety applications. Analytical and numerical investigation of V2I, $\mathrm{V} 2 \mathrm{~V}$ networks and their applications have been a subject of intense investigation for a good part of the past decade [1]. The theoretical performance of the medium access control (MAC) for beacon transmissions are investigated in [3] - [5]. The research efforts on MAC, however, did not consider the PHY and channel characteristics, including the impact of slow and fast fading as well as interference coming from beyond the sensing range. Moreover, the road traffic distribution is often not taken into account. Radio propagation characteristics of the IEEE 802.11p has been investigated in measurement campaigns [6] - [8]. Cheng et al. [6] introduced power loss models that are fitting into their measurement results. Such an approach is well motivating when one considers transmission of information over a long period of time, i.e. for continuous data traffic but not necessarily for transmission of short messages, e.g. beacons. We believe that a more accurate fading model, one which includes fast fading and frequency selectivity of an outdoor sideband channels, is required in studying beacon performance. Moreover, the existing work on PHY considers a single V2X link ignoring the behavior of MAC, or more precisely, interference created by other vehicles.

This paper investigate the problem of performance of packet delivery in the IEEE $802.11 \mathrm{p}$ vehicular network, with emphasis on the effects of the wireless channel in combination with MAC protocol and some parameters of car traffic. The major contributions of the paper are

- Comparing with experimental results, we show that the dual slope linear path model well approximates average signal quality of vehicular communications. An important insight achieved from the study is that in addition to the distance dependent pathloss, it is necessary to consider the fading effects, which results in fluctuation of signal quality. To address this issue, the effect of power delay profile is studied.

- The interference induced from simultaneous transmissions is analyzed considering MAC behavior and realistic traffic densities. Traffic density is modelled with Poisson and Lattice distributions.

- Using computer simulations, the PDR performance is investigated and show that the MAC behavior and the realistic traffic parameters affect the system performance in such a way that the effective communication range largely degrades with the increase of the road density.

The paper is organized as follows. Section II reviews a few analytical models of fading channels relevant for V2X scenarios. This includes extraction of parameters from the Received Signal Strength Indicator (RSSI) measurements including accounting for fast fading and frequency selectivity. Section III contains calculations of Signal-to-Interference plus Noise ratio (SINR) in a receiving node, which accounts for both power loss and fading due to propagation channel as well as interference created by the other vehicles on the road. The calculation of interference is based on two realistic models of distribution of cars for different driving conditions (car density). Section IV provides detailed analytical treatment of modulation performance on the PHY layer. Furthermore, Section V links PHY level parameters with those of MAC layer. Finally, we provide some conclusions.

\section{Channel Model}

\section{A. Path loss model derived from experimental data}

Signal quality is the key characteristics to determine the communications performance including PDR, delay, and 
throughput. It is common to express the received signal quality for a given frequency $\omega$ by the distance-dependent path loss, large-scale fading, and small scale fading:

$$
P(\omega, d)=P\left(\omega, d_{0}\right)-10 n \log _{10}\left(d / d_{0}\right)+X_{\sigma}(\omega)+Y(\omega)
$$

where $P(\omega, d)$ is the power level in $\mathrm{dBm}$ measured at the frequency $\omega$ at the distance $d$ from the source; $n$ is path loss exponent, $X_{\sigma}(\omega)$ and $Y(\omega)$ are large and small scale fading components. Often, $X_{\sigma}(\omega)$ is taken to be Gaussian, corresponding to log-normal large scale fading, and $Y(\omega)$ corresponds to Rayleigh, Nakagami, Rice fading [9].

Because the distance-dependent pathloss provides the key impact on the average signal quality, it is necessary to have correct understanding of its characteristics in the DSRC $5.9 \mathrm{GHz}$ band.

The simplest pathloss model is the free space model that describes the line of sight (LoS) ray propagation:

$$
L_{\text {free }}[\mathrm{dB}]=20 \log _{10}\left(4 \pi \frac{d}{\lambda}\right),
$$

where $\lambda$ is the wavelength. A more appropriate model is the Two-Ray Ground reflection model, which takes into account the ground reflected ray in addition to the direct LoS ray:

$$
L_{t r g}[\mathrm{~dB}]=40 \log _{10} d-20 \log _{10}\left(h_{t} h_{r}\right),
$$

where $h_{t}$ and $h_{r}$ are the transmit and receive antenna heights respectively. Yet another model to characterize the pathloss is the dual slope linear model, which takes the following form [6]

$$
P(d)= \begin{cases}P_{\text {near }}(1)-10 \gamma_{\text {near }} \log _{10}(d) & \text { if } d \leq d_{c} \\ P_{\text {far }}(1)-10 \gamma_{\text {far }} \log _{10}(d) & \text { if } d>d_{c}\end{cases}
$$

Here $P_{\text {near }}(1)$ and $P_{\text {far }}(1)$ are constant values; $d_{c}$ is the extent of the Fresnel zone

$$
d_{c}=4 h_{t} h_{r} M / \lambda
$$

and $\gamma_{\text {near }}$ and $\gamma_{\text {far }}$ describe slope of the attenuation in the near and the far zones.

In order to evaluate the appropriateness of the models, we compare the curves provided by the pathloss models to the results obtained from the real-world experiments, which have been introduced in [7]. The experiments are carried out on a $1.6 \mathrm{~km}$ line of sight straight road, where a road side unit (RSU) is installed at 400 meters from one end of the road. A vehicle runs from this end to the other end of the road. Both the RSU and the vehicle are equipped with the $802.11 \mathrm{p}$ communications devices, whose antenna heights are 3 and 1.5 meters, respectively. The RSU broadcasts 200-bytes of messages, generated at $2 \mathrm{Mbps}$, at a transmission power of $24 \mathrm{dBm}$. The received signal quality, RSSI, is measured at the vehicle. The speed of the vehicle takes on different values for different runs. The above mentioned pathloss models and the experimental results are compared in Figs. 1 - 2. The figures show that although the two-ray model provides a good approximation for small distances, the dual slope linear model shows better approximation especially for large distances. The

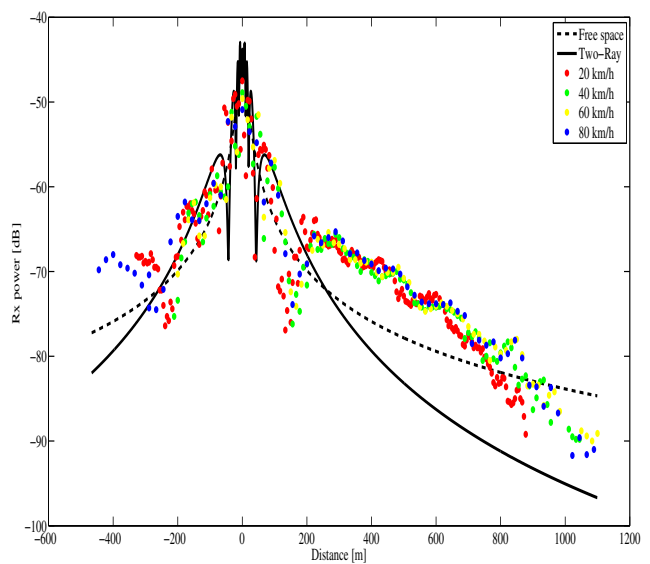

Fig. 1. Approximition using free space and two-ray models (Transmission rate is fixed to $3 \mathrm{Mbps}$ ).

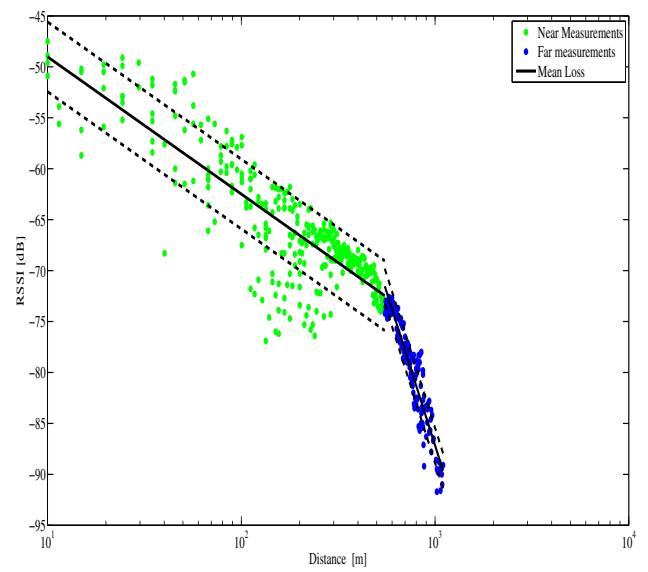

Fig. 2. Approximation using Log-Normal distribution. $\gamma_{\text {near }}=1.16, \gamma_{\text {far }}=$ 5.4 (The transmission rate and speed are same as those in Fig. 1).

individual RSSI values, however, are largely spread around the average value. A similar observation can also be made from the PDR vs. RSSI measurement results shown in Fig. 3. The observations indicate the need for considering the fading effects in greater detail.

\section{B. Effects of Power Delay Profile}

All wideband systems experience frequency selective fading, due to multipath propagation and non-delta shaped power delay profile (PDP) $P(\tau)$ [10]. This will cause a number of performance degrading consequences, such as inter symbol interference (ISI), inter channel interference (ICI), unequal performance over $N_{c}$ different subcarriers, etc. While adding $N_{c p}$ symbol cyclic prefix eliminates majority of ISI, some remnant of ISI and induced ICI (due to loss of carrier orthogonality among sub-carriers) remains and reduces performance of OFDM. While the total power of OFDM transmission is captured and can be measured in testbed systems as RSSI (see 


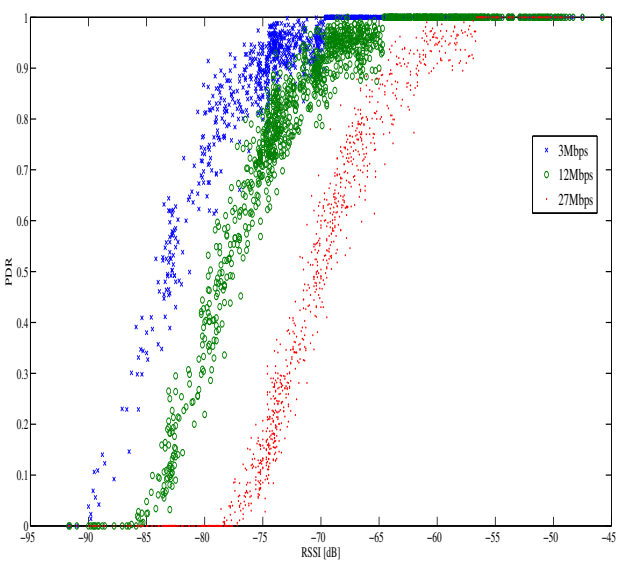

Fig. 3. Packet delivery ratio (PDR) as function of RSSI.

Figs. 1 - 3), more refined characteristics are hidden from it. This is why the PDR vs RSSI graphs, have number of artifacts as can be seen in Fig. 3 .

One approach to improve usability of RSSI as a channel quality factor is to define the effective SNR $\gamma_{e f f, \mathrm{M}}$ [11] such that for a given modulation type $M$

$$
N_{c} P_{b}\left(\gamma_{e f f, \mathrm{M}}\right)=\sum_{k=1}^{N_{c}} P_{b}\left(\gamma_{k}\right)
$$

In this case, the same BER $P_{b}$ is achieved both in frequency selective fading and the equivalent flat fading with SNR $\gamma_{e f f, M}$. Importantly, the effective SNR depends on the modulation used. However, corrective factors in [11] are based solely on simulations and are not related to the shape of PDP.

More insight could be achieved by applying derivations, presented in [12]: the effective SNR is degraded, comparing to $E_{0} / N_{0}$ as measured by RSSI, by the following factor

$$
\gamma_{d e g}=-10 \log _{10}\left(\frac{\frac{N_{c}}{N_{c}+N_{c p}} p_{u}}{1+\gamma_{\frac{N_{c}}{N_{c}+N_{c p}}}\left(1-p_{u}\right)}\right)
$$

Here $p_{u}$ is a fraction of the power, measured by RSSI, which associated with the information symbol, while $\left(1-p_{u}\right)$ is a fraction of power associated with ISI and ICI, caused by multipath and cyclic prefix use as calculated in [12]. Even if ICI and ISI are removed by means of the cyclic prefix, the frequency selectivity results into random variations of SNR and BER in different sub-channels. Let $\bar{\gamma}$ be average value of SNR in each individual sub-carrier and $\gamma=\left[\gamma_{1}, \gamma_{2}, \cdots, \gamma\right]$ be a vector of instantaneous SNR on each subcarrier. Distribution of $\gamma$ is given by multidimensional correlated Gamma PDF [9]. We leave details out due to the lack of space. In the limiting case, when $P(\tau)=\delta\left(\tau-\tau_{0}\right)$, the fading is frequency flat, all components of the vector $\gamma$ are completely correlated, i.e. all subcarriers have the same value at any given time instant. There is no selectivity and RSSI directly reflects SNR on each subcarrier.
Another extreme case is when the spread of PDF is so significant, that the channel gains at the frequencies corresponding to each subcarrier vary independently. In this case, measurement of RSSI corresponds to accumulation of random powers at each sub-channel and the noise

$$
R S S I=N_{c} \tilde{\gamma}+\sigma_{\xi}^{2}=\sum_{n=1}^{N_{c}} \gamma_{i}+\sigma_{\xi}^{2}
$$

The probability density of vector $\gamma$, given measured RSSI, can be given by

$$
p(\gamma, \tilde{\gamma})=\delta\left(N_{c} \tilde{\gamma}-\sum_{n=1}^{N_{c}} \gamma_{n}\right) p_{N_{c}}\left(\gamma_{1}, \gamma_{2}, \cdots, \gamma_{N_{c}}\right)
$$

Thus, the effective BER, defined in (6) could be expressed as

$$
P_{b}\left(\gamma_{b, \mathrm{M}}, \tilde{\gamma}\right)=\frac{1}{N_{c}} \sum_{k=1}^{N_{c}} E\left\{P_{b}\left(\gamma_{k}\right)\right\}=E\left\{P_{b}\left(\gamma_{1}\right)\right\}
$$

Here the expectation is taken over the marginal distribution $p_{1}\left(\gamma_{1}\right)$, obtained by integrating (9) over auxilirary variables $\gamma_{2}, . ., \gamma_{N_{c}}$. Once again, leaving detailed calculations to further publications, we show the final result

$$
p_{1}\left(\gamma_{1}\right)=\frac{\left(N_{c} \tilde{\gamma}-\gamma_{1}\right)^{N_{c}-1}}{\tilde{\gamma}\left(N_{c} \tilde{\gamma}\right)^{N_{c}-1}}, \quad 0 \leq \gamma_{1} \leq N \tilde{\gamma}
$$

In the case of beacons, the modulation is BPSK, $P_{b}(\gamma)=$ $Q(\sqrt{2 \gamma})$ and the following approximation could be obtained:

$$
P_{b}\left(\gamma_{e f f}, B P S K\right) \approx Q(\sqrt{2 \tilde{\gamma}})+\frac{\sqrt{\tilde{\gamma}} \exp (-\tilde{\gamma})}{\sqrt{\pi}\left(N_{c}+1\right)}
$$

which gives an upper bound on degradation of SNR due to PDP. We leave detailed consideration for further publications.

\section{EVALUATION OF SIR AND SINR}

The packet delivery performance between a given transmitter and receiver is largely affected by the interference coming from the simultaneously transmitting nodes. While MAC's role is to schedule transmissions such that interference is avoided, simultaneous transmissions still can happen due to 1) hidden terminal problem (i.e., a node beyond the sensing range transmits) and 2) the small contention window size, which leads nodes transmit at the same time even they are within each others sensing range. Because the latter generally causes "packet collision", we believe it is sufficient to formulate using the MAC level packet collision analysis (which will be discussed in Section V). The former is, however, does not necessary result in "collision", therefore SIR has to be investigated.

An important consideration in the study of SIR is the relative location of the network nodes. An accurate assessment of V2X network performance thus requires an understanding of how vehicular traffic behaves. Often, parameters of car traffic could be extracted from measurements as discussed in [13] by fitting distribution of the headway time headway and velocity 


\begin{tabular}{|l|c|c|c|c|c|}
\hline Modulation & $\begin{array}{c}\text { Code } \\
\text { rate } \mathbf{C}_{\mathbf{R}}\end{array}$ & $\begin{array}{c}\text { Data } \\
\text { rate R }\end{array}$ & $\mathbf{d}_{\text {free }}$ & $\begin{array}{c}\text { Minimum } \\
\text { Sensitivity }\end{array}$ & $\begin{array}{c}\text { Threshold } \\
\text { SNR, dB }\end{array}$ \\
\hline BPSK & $\mathbf{1 / 2}$ & $\mathbf{3}$ & $\mathbf{1 0}$ & $-85 \mathrm{dBm}$ & $<10$ \\
BPSK & $3 / 4$ & 4.5 & 5 & $-84 \mathrm{dBm}$ & 10 \\
QPSK & $1 / 2$ & 6 & 10 & $-82 \mathrm{dBm}$ & 11 \\
QPSK & $3 / 4$ & 9 & 5 & $-80 \mathrm{dBm}$ & 14 \\
\hline
\end{tabular}

SUMMARY OF IEEE802.11P PHY LAYER PARAMETERS

measurements. The exponential distribution (therefore, the Poisson model of number of cars on $l$ interval)

$$
p(l)=\lambda \exp (-\lambda l)
$$

is used in [14], as being particularly accurate in a low density traffic conditions. A Pearson type III [14] and the generalized extreme value distribution is used in [15] to approximate real time measurements in different driving conditions. In this paper we focus on two limiting cases of Pearson type III distribution to model distribution of cars on a road: Poisson process with exponential of inter-car distances (suitable for light traffic) and lattice distribution with constant inter-car distance which is representative of heavy road.

\section{A. Poisson distribution of cars}

Let us consider Poisson distribution of cars with density $\lambda$ (cars/meter), which is a good model for relatively thin traffic on a highway. Let $\tau$ be channel access probability by an individual car ( $\tau$ will be formulated in Section $\mathrm{V})$ and $r$ be a distance between the receiving car and interfering car. Let us consider a short interval $d r$ centered at the distance $r$ from the receiver. Then, the interference power induced at the receiver is given by

$$
d P_{I}(r)=P_{T 0} \tau\left(\frac{r}{d_{0}}\right)^{-\alpha} \lambda d r
$$

Here $P_{T 0}$ is the transmitter power measured at the distance $d_{0}$ from the antenna, and $\alpha$ is the path loss exponent. Integrating over complete interference range we can determine the average interference power as

$$
P_{I}=\int_{R}^{\infty} d P_{I}(r)=\lambda \tau R P_{T 0}\left(\frac{d_{0}}{R}\right)^{\alpha}
$$

Here $R$ is the sensing range.

\section{B. Lattice distribution of cars}

If traffic is quite dense, a lattice model of car distribution could be more appropriate. In this case the distance between any two cars has a fixed value $\bar{d}=1 / \lambda$. The $n$-th vehicle from the fringe of the reception zone is thus at the distance $R+n \bar{d}$ from the receive node. Thus, the interference power at the receiver due to this node, is given by

$$
P_{T, n}=\tau P_{T 0}\left(\frac{\bar{d}}{d_{0}}\right)^{-\alpha} \frac{1}{(R / \bar{d}+n)^{\alpha}}
$$

Therefore, the average interference power at the receiver is

$$
P_{I}=\sum_{n=0}^{\infty} P_{T, n}=\tau P_{T 0}\left(\frac{\bar{d}}{d_{0}}\right)^{-\alpha} \zeta(\alpha, R / \bar{d}+1)
$$

Here $\zeta(m, x)$ is Hourwitz zeta function [16].

\section{SIR calculation}

Let a transmitting node be located at the distance $r$ from the tagged node at $r=0$. In this case the received power is given by

$$
P_{s}(r)=P_{T 0}\left(\frac{r}{d_{0}}\right)^{-\alpha}
$$

Therefore, the SIR ratio at the receiver is given by

$$
S I R(r)=\frac{P_{s}}{P_{I}}=\frac{1}{\lambda \tau R}\left(\frac{R}{r}\right)^{\alpha}
$$

in the case of Poisson car distribution, and, in the case of lattice distribution, by

$$
\operatorname{SIR}(r)=\frac{1}{\tau \zeta(\alpha, R / \bar{d}+1)}\left(\frac{\bar{d}}{r}\right)^{\alpha}
$$

Coupled with SNR, measured or calculated from propagation models, the resulting SINR value could be calculated as

$$
\mathrm{SINR}^{-1}=\mathrm{SNR}^{-1}+\mathrm{SIR}^{-1}
$$

Furthermore, this equations could be used to calculate the probability of error of packed detection and in evaluation of the receive and the sensing ranges. Indeed, the sensing range could be defined as the minimum distance at which the interference power given by equations (15) or (17) exceeds the noise floor of the receiver, as calculated below in (22). The receive range, for a given modulation scheme, can then be evaluated as the maximum distance at which the SINR exceeds the sensitivity of the modulation, as described by IEEE $802.11 \mathrm{p}$ and listed in the Table I. It is important to note that the values of receive and sensing ranges are functions of node transmit power, density of the nodes, their statistics and the transmission policy at the MAC layer. Similar calculations could be conducted in the case of power loss law approximated by two slope model.

\section{Modulation, DATA RATE AND CODING}

The IEEE 802.11p standard [17] defines PHY layer frame as consisting of PLCP preamble, $32 \mu$ s long for $10 \mathrm{MHz}$ WAVE channels, SIGNAL field which is $8 \mu$ s long (one OFDM symbol) and sequence of OFDM data symbols. Table I contains brief summary of parameters of the BPSK and QPSK modulations of PHY level as outlined in [17]. Minimum receiver sensitivity is defined at the level of PER of $10 \%$ for PSDU length of 1000 octets (bytes). Noise factor of $10 \mathrm{~dB}$ and $5 \mathrm{~dB}$ implementation margins are assumed [17].

The background noise of the channel can be calculated according to the Johnson's formula

$$
P_{n, d B m}=10 \log _{10}\left(1000 k_{B} T \Delta F\right) \approx-174+10 \log _{10}(\Delta F)
$$


For IEEE802.11p channel $\Delta F=10^{7} \mathrm{~Hz}$ and, therefore, $P_{n, d B m}=-104 \mathrm{dBm}$. Adding another $5 \mathrm{~dB}$ to account for the device noise, the noise floor is of order of $-99 \mathrm{dBm}$.

In terms of per bit SNR $\gamma=E_{b} / N_{0}$ probability of bit error $P_{b}$ for BPSK and QPSK can be calculated as

$$
P_{b}=Q(\sqrt{2 \gamma})
$$

Similar expressions could be derived for higher order modulation [9], which we omit since it is not used for beacon distribution.

The IEEE $802.11 \mathrm{p}$ mandates use of convolutional codes with industry standard generator polynomials $G_{0}=133_{8}$ and $G_{I}=171_{8}$, constarin length $K=7$, to obtain mother coded sequence of the code rate $C_{R}=1 / 2$ followed by pucturing to obtaine rates $C_{R}=2 / 3$ and $C_{R}=3 / 4$. Decoding by Viterbi algorithm is recommended. Total number $a_{d}$ of errer events of hamming distance $d$ from the correct path can be obtained either from the transfer function or by a numerical search.

Specific of V2X for safety applications is that exchange of information between devices is in a form of small amounts of data. Thus, communication session last only a short period of time, consequently it is exposed only to a single value of fading. Therefore, outage probability of packet reception is a proper measure of performance. It is difficult to account for effect of coding and calculate packet error rate (PER). It is suggested in a number of publications (see [18] and references within) that upper bound of PER $P_{P E R}(L)$ could be used

$$
P_{P E R}(L) \leq 1-\left(1-P_{u}\right)^{8 L}
$$

where $L$ is the packet size in bytes and $P_{u}$ is the union bound of the first event error probability given by

$$
P_{u}=\sum_{d=d_{\text {free }}}^{\infty} a_{d} \cdot P_{d}
$$

The free distance $d_{\text {free }}$ of the code is shown in Table I, $a_{d}$ is the total number of errors with weight $d$ and $P_{d}$ is the probability of error in the pairwise comparison of two path differ in $d$ bits. The values of $a_{d}$ (weighted spectra) could be found as coefficients of expansion of code transfer function into power series and represent number of path of distance $d$ from all-zero path summarized in Table II. When hard-decision decoding is used, $P_{d}$ can be calculated as

$$
P_{d}= \begin{cases}\sum_{k=\frac{d+1}{2}}^{d}\left(\begin{array}{c}
d \\
k
\end{array}\right) p^{k}(1-p)^{d-k} & d=\text { odd } \\
\sum_{d / 2+1}^{d} p^{k}(1-p)^{d-k}+ & \\
\frac{1}{2}\left(\begin{array}{c}
d \\
d / 2
\end{array}\right) p^{d / 2}(1-p)^{d / 2} & d=\text { even }\end{cases}
$$

\section{MAC MODEL AND SIMULATIONS}

The IEEE 802.11p adopts the Enhanced Distributed Channel Access (EDCA), defined in the IEEE 802.11e to provide differentiated channel access to transmissions of packets belonging to different access categories (ACs). In this paper, however, we model the channel access probability for a single AC

\begin{tabular}{|l|l||l|l|l|}
\hline$\rho$ & $d_{\text {free }}$ & \multicolumn{1}{c|}{$\begin{array}{c}d \\
a_{d}\end{array}$} & \multicolumn{1}{c|}{\begin{tabular}{c}
\multicolumn{1}{c|}{$a_{d}$} \\
$a_{d}$
\end{tabular}} \\
\hline $1 / 2$ & 10 & $\begin{array}{l}10 \\
11 / 14\end{array}$ & $\begin{array}{l}12 \\
38 / 14\end{array}$ & $\begin{array}{l}14 \\
193 / 14\end{array}$ \\
\hline $2 / 3$ & 6 & $\begin{array}{l}6 \\
1 / 14\end{array}$ & $\begin{array}{l}7 \\
16 / 14\end{array}$ & $\begin{array}{l}8 \\
48 / 14\end{array}$ \\
\hline $3 / 4$ & 5 & $\begin{array}{l}5 \\
8 / 14\end{array}$ & $\begin{array}{l}6 \\
31 / 14\end{array}$ & $\begin{array}{l}7 \\
160 / 14\end{array}$ \\
\hline
\end{tabular}

TABLE II

WEIGHT SPECTRA COEFFICIENTS $a_{d}$ FOR IEEE802.11P STANDARD

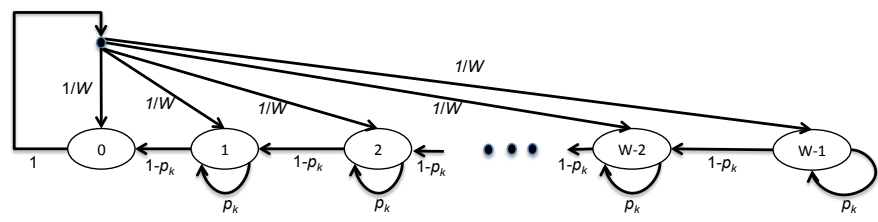

Fig. 4. Markov chain as a model for MAC layer

for broadcast frames (e.g., beacons) using one dimensional Markov chain illustrated in Fig. 4.

We first model for saturated conditions, and extend our model for more general cases. The state $b(i)$ of the Markov chain represents the countdown states. $p_{k}$ is the channel blocking probability, i.e., the probability of channel being busy due to activities at the nodes other than the tagged node (i.e., the node which is the subject to the Markov chain) and $W$ is minimum contention window size for the AC. Solving the Markov chain, i.e., $\sum_{i=0}^{W-1}(i)=1$, the channel access probability for saturated conditions is found as

$$
\tau_{s}=b(0)=\left[1+\frac{W-1}{2\left(1-p_{k}\right)}\right]^{-1} .
$$

The correctness of the model can be verified by comparing it to the well known Bianchi model [19]. In the Bianchi model, $p_{k}$ is not taken into account. Therefore, by setting $p_{k}$ to 0 , equation (26) delivers $\tau=2 /(W+1)$ coinciding with the result of the Bianchi model [19].

The channel access probability for general channel conditions can be formulated as:

$$
\tau=q \times \tau_{s},
$$

where $q$ is the probability of a pending packet at the node. Assuming messages are generated following the Poisson process, the probability of a pending packet is

$$
q=1-\exp \left(-\lambda Y_{s}\right) .
$$

Here, $\lambda$ is the frame generation rate, $Y_{s}$ is the average channel service time. Letting $T$ be the time required for a transmission of a frame (including the arbitrary inter-frame space, AIFS), and $\sigma$ be the slot time, the average channel service time is

$$
Y_{s}=p_{b} T+\left(1-p_{b}\right) \sigma
$$

where $p_{b}$ is the probability of busy channel. Because the tagged node cannot sense the signal from a transmitting node, 


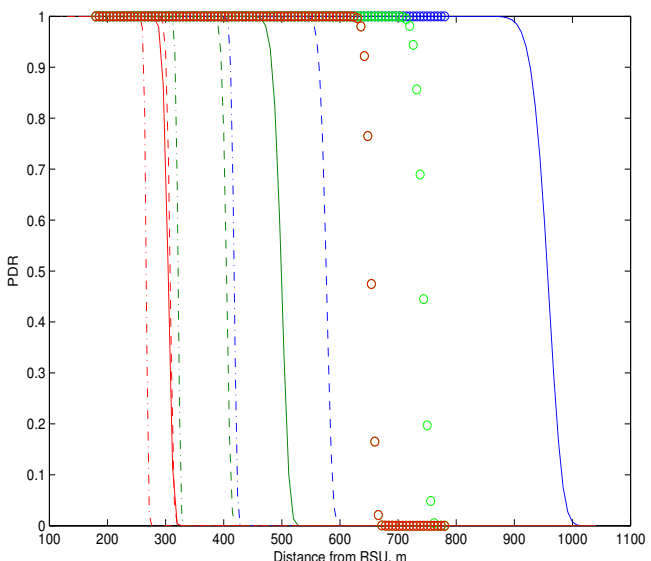

Fig. 5. PDR as function of the receiving vehicular. Solid line $\alpha=2$, dashed line $\alpha=3$, dashed-dotted line $\alpha=4$. Blue -3 cars $/ \mathrm{km}$, green $-25 \mathrm{cars} / \mathrm{km}$, red - $80 \mathrm{cars} / \mathrm{km}$. Circles correspond to evaluation based on the experimental data as described in Section II.

which is beyond its sensing range, $\mathrm{R}$, we can consider only the nodes in the sensing range for calculation of $p_{b}$ and $p_{k}$ :

$$
p_{b}=1-(1-\tau)^{N_{R}} p_{k}=1-(1-\tau)^{N_{R}-1}
$$

where $N_{R}$ is the number of nodes in the sensing range. It is worth mentioning, that (27)-(30) form a system of equations which must be solved simultaneously.

In order to evaluate PDR, one has to note, that a beacon if transmitted, is successfully delivered if it is transmitted without a collision and properly decoded. In other words

$$
P D R=(1-\tau)^{\left(N_{R}-1\right)} \cdot\left(1-P_{P E R}\right)
$$

where $P_{P E R}$ is the packet error rate as given by eq. (23).

To show the effect of the interference from multiple cars on the road, we conduct simulation of PDR as a function of the receiver's distance from the transmitter, path loss exponent and car density. The results are shown in Fig. 5. It can be seen that for a very thin traffic, the transmission range coincides with that obtained from single Tr-Rx measurements. However, once the density increases, the transmission range shrinks which adversely affects the PDR. This effect is especially pronounced for small values of $\alpha$, pathloss exponent.

\section{CONCLUSIONS}

We have considered evaluation of delivery of beacons in V2X networks, based on a single link RSSI measurements. We have shown how fast fading effects could lead to spread of experimental values in PDR vs RSSI plots as in Fig. 3. We also have shown how PDP of frequency selective fading could be accounted for proper bit error calculations as well as how to account for traffic density. It can be seen, that various car densities have profound effect on the range of beacon delivery, especially for small values of path loss exponent.

\section{REFERENCES}

[1] M. Javadi, S. Habib, and M. Hannan, "Survey on inter-vehicle communication applications: Current trends and challenges," Information Technology Journal, vol. 12, no. 2, pp. 243-250, 2013.

[2] IEEE Standard for Information technology - Telecommunications and information exchange between systems - Local and metropolitan area networks - Specific requirement, Part 11: Wireless LAN Medium Access Control (MAC) and Physical Layer (PHY) Specifications, IEEE Computer Society Std., July 2010, iEEE Std 802.11p-2010.

[3] A. Vinel, Y. Koucheryavy, S. Andreev, and D. Staehle, "Estimation of a successful beacon reception probability in vehicular ad-hoc networks," in Proceedings of IWCMC, 2009, pp. 416-420.

[4] X. Chen, H. Refai, and X. Ma, "Saturation performance of IEEE 802.11 broadcast scheme in ad hoc wireless LANs," in VTC-2007 Fall, 2007, pp. 1897-1901.

[5] X. Ma and X. Chen, "Delay and broadcast reception rates of highway safety applications in vehicular ad hoc networks," in 2007 Mobile Networking for Vehicular Environments, 2007, pp. 85-90.

[6] L. Cheng, B. Henty, D. Stancil, F. Bai, and P. Mudalige, "Mobile vehicle-to-vehicle narrow-band channel measurement and characterization of the $5.9 \mathrm{GHz}$ dedicated short range communication (DSRC) frequency band," IEEE J. Sel. Areas Commun., vol. 25, no. 8, August 2007.

[7] O. Shagdar, M. Tsukada, M. Kakiuchi, T. Toukabri, and T. Ernst, "Experimentation Towards IPv6 over IEEE 802.11p with ITS Station Architecture," in International Workshop on IPv6-based Vehicular Networks (colocated with IEEE Intelligent Vehicles Symposium), June 2012.

[8] J.-C. Lin, C.-S. Lin, C.-N. Liang, and B.-C. Chen, "Wireless communication performance based on IEEE 802.11p R2V field trials," IEEE Commun. Mag., vol. 50, no. 5, pp. 184-191, May 2012.

[9] M. Simon and M.-S. Alouini, Digital Communication over Fading Channels. New York: John Wiley \& Sons, 2000.

[10] A. Papoulis, Probability, Random Variables, and Stochastic Processes, 3rd ed. Boston, MA: McGraw-Hill, 1991.

[11] D. Halperin, W. Hu, A. Sheth, and D. Wetherall, "Predictable 802.11 packet delivery from wireless channel measurements," in Proceedings of the ACM SIGCOMM 2010 conference, ser. SIGCOMM '10. New York, NY, USA: ACM, 2010, pp. 159-170.

[12] H. Steendam and M. Moeneclaey, "Analysis and optimization of the performance of OFDM on frequency-selective time-selective fading channels," IEEE Trans. Commun., vol. 47, no. 12, pp. 1811-1819, 1999.

[13] B. Kerner, The Physics of Traffic: Empirical Freeway Pattern Features, Engineering Applications, and Theory, ser. Springer Complexity. Springer, 2004.

[14] A. May, Traffic Flow Fundamentals. Prentice Hall, 1990.

[15] L. Cheng and S. Panichpapiboon, "Effects of intervehicle spacing distributions on connectivity of VANET: a case study from measured highway traffic," IEEE Commun. Mag., vol. 50, no. 10, pp. 90-97, 2012.

[16] M. Abramowitz and I. Stegun, Eds., Handbook of Mathematical Functions. New York: Dover, 1965.

[17] IEEE802.11, "IEEE Standard for Information Technology - Telecommunications and Information Exchange Between Systems - Local and Metropolitan Area Networks - Specific Requirements - Part 11: Wireless LAN Medium Access Control (MAC) and Physical Layer (PHY) Specifications," IEEE Std 802.11-2007 (Revision of IEEE Std 802.11-1999), pp. $1-1076,122007$.

[18] Y. Zhang, L. Stibor, G. Orfanos, S. Guo, and H.-J. Reumerman, "An error model for inter-vehicle communications in highway scenarios at $5.9 \mathrm{GHz}$,' in PE-WASUN '05, 2005, pp. 49-56.

[19] G. Bianchi, "Performance analysis of the IEEE 802.11 distributed coordination function," Selected Areas in Communications, IEEE Journal, vol. 18 , no. 3 , pp. 535-547, 2000 